\title{
Samuel Rufat et Hovig Ter Minassian (dir.), Les jeux vidéo comme objet de recherche
}

\section{Henri Desbois}

\section{(2) OpenEdition}

1 Journals

Édition électronique

URL : http://journals.openedition.org/cdg/2789

DOI : $10.4000 /$ cdg. 2789

ISSN : 2107-7266

Éditeur

UMR 245 - CESSMA

Référence électronique

Henri Desbois, « Samuel Rufat et Hovig Ter Minassian (dir.), Les jeux vidéo comme objet de recherche », Carnets de géographes [En ligne], 2 | 2011, mis en ligne le 02 mars 2011, consulté le 24 septembre 2020. URL : http://journals.openedition.org/cdg/2789; DOI : https://doi.org/10.4000/cdg.2789

Ce document a été généré automatiquement le 24 septembre 2020.

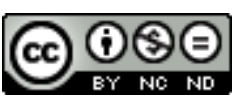

La revue Carnets de géographes est mise à disposition selon les termes de la Licence Creative Commons Attribution - Pas d'Utilisation Commerciale - Pas de Modification 4.0 International. 


\title{
Samuel Rufat et Hovig Ter Minassian (dir.), Les jeux vidéo comme objet de recherche
}

\author{
Henri Desbois
}

\section{RÉFÉRENCE}

Les jeux vidéo comme objet de recherche Samuel Rufat et Hovig Ter Minassian (dir.)

L>P Questions Théoriques, 2011

1 Les jeux vidéo comme objet de recherche est un ouvrage collectif issu des travaux du laboratoire junior «Jeux vidéo, pratiques, contenus, discours». Il regroupe 11 contributions précédées d'une introduction. Une des originalités de ce recueil est de réunir à la fois des auteurs de diverses sciences humaines et des acteurs de l'industrie du jeu vidéo, notamment des concepteurs de «jeux sérieux ». Ce mélange est parfois déconcertant. Il est assez inhabituel de tomber sur des lignes de code en $\mathrm{C}$ dans un ouvrage plutôt situé dans le champ des sciences humaines (c'est le cas pour un seul article et cela ravira, n'en doutons pas, certains lecteurs friands de technique). Les articles sont regroupés selon quatre thèmes : la question de la conception, la question de l'espace dans le jeu, envisagé à la fois du point vue théorique et à partir d'exemples, les usages, notamment pédagogique du jeu, et les pratiques, avec une approche par le langage et la sociologie. Ce regroupement cependant recouvre une telle diversité d'approches et de sujets, même à l'intérieur de chaque thème, qu'on oublie souvent, à la lecture, à quel thème appartient chaque texte.

2 C'est cette diversité d'approches qui fait la richesse de l'ouvrage, dans lequel la plupart des personnes intéressées à un titre ou à un autre par les jeux vidéo pourront trouver quelque chose. En revanche, le caractère éclaté et la spécialisation souvent assez marquée des différents articles ne permet pas de recommander le livre à ceux qui chercheraient une introduction à ce domaine. Le titre est bien conforme au contenu du 
livre. Il s'agit d'un panorama extrêmement divers des «jeux vidéo comme objets de recherche ». L'ouvrage est relativement bref (environ 200 pages), et ne couvre pas tous les aspects de jeux vidéo (par exemple, il n'y a pas d'article sur l'économie du secteur), mais l'exhaustivité serait impossible même pour un livre beaucoup plus volumineux. Il est dommage qu'il n'y ait ni index des notions ni des jeux cités, ce qui aurait permis de naviguer plus aisément entre les textes.

Les géographes seront plus particulièrement intéressés par l'article des deux directeurs du recueil : «Espace et jeux vidéo », qui propose une étude très documenté sur l'espace dans (et autour) des jeux vidéo. Le chapitre sur les représentations de l'antiquité dans les jeux vidéo, écrit conjointement par deux auteurs, l'une spécialiste d'esthétique, l'autre de l'antiquité, a également une forte dimension spatiale et retiendra l'attention des géographes intéressés par le thème du paysage.

D'une manière générale, à l'exception peut-être des textes produits par des acteurs du jeu vidéo, la plupart des articles de ce recueil sont plus ou moins animés par une attitude presque militante en faveur de la reconnaissance du jeu vidéo comme objet de recherche légitime dans l'université, voire comme outil ou support pédagogique. Peutêtre faut-il voir là un effet de génération comparable à celui qui avait fait entrer la science-fiction dans les universités américaines dans les années 1970.

5 Si cet ouvrage démontre de façon convaincante comment les disciplines universitaires constituées peuvent s'approprier l'objet vidéoludique, il est trop tôt pour dire si l'étude des jeux vidéo en elle-même peut et doit se constituer en discipline autonome. Parmi les questions que le livre laisse ouvertes, celle de la place du jeu vidéo dans un système plus global de cultures numériques et d'industrie des loisirs en général est sans doute une des plus importantes. On ne peut que souhaiter que l'équipe de recherche à l'origine du présent ouvrage poursuive et complète ses travaux dans les années qui viennent.

\section{INDEX}

Thèmes : Carnets de lectures

\section{AUTEURS}

\section{HENRI DESBOIS}

Maître de conférences

Géographe

Université Paris Ouest Nanterre la Défense 